\title{
A RICH, NEARBY GALAXY CLUSTER IN SAGITTARIUS ${ }^{a)}$
}

\author{
S. Djorgovski, ${ }^{\text {b),c) }}$ D. J. Thompson, R. R. De Carvalho, and J. R. Mould \\ Palomar Observatory, Division of Physics, Mathematics, and Astronomy, California Institute of Technology, Pasadena, California 91125 \\ Received 20 March 1990; revised 5 June 1990
}

\begin{abstract}
We report on the existence of a rich, heavily obscured galaxy cluster (or a set of clusters) in Sagittarius, centered near $l_{\mathrm{II}} \simeq 359^{\circ}, b_{\mathrm{II}} \simeq 8^{\circ}$. About 30 objects, originally identified by Terzan and his collaborators, were imaged and followed up spectroscopically. We present here redshifts for 21 galaxies found among them. There is a pronounced concentration at $c z \simeq 8600 \mathrm{~km} / \mathrm{s}$ (containing 14 out of the 21 galaxies), and another possible concentration near $c z \simeq 11340 \mathrm{~km} / \mathrm{s}$ (five out of 21 ). This putative Sagittarius cluster is probably part of a larger system, involving the Ophiuchus cluster at $l_{\mathrm{II}} \simeq 1^{\circ}, b_{\mathrm{II}} \simeq 9^{\circ}, c z \simeq 8400$ $\mathrm{km} / \mathrm{s}$, previously identified by Johnston et al. and Wakamatsu and Malkan. The heavy extinction on this line of sight (we estimate $A_{V} \sim 5^{\mathrm{m}}$ from the observed Balmer decrements) suggests that there is a much larger, as yet undetected number of galaxies in this direction. We propose that the SagittariusOphiuchus concentration may be a massive cluster, or even a supercluster, comparable in richness to the Coma-A1367 system, and at a comparable distance. The existence of this concentration serves as a reminder that some possibly dynamically important constituents of the local large-scale structure may be absent from the galaxy catalogs available now, and the dynamical analyses based on them.
\end{abstract}

\section{INTRODUCTION}

Study of the large-scale structure is one of the most active topics of research in extragalactic astronomy today, yet all of the existing galaxy and cluster catalogs which are used for most of the studies are severely incomplete at low Galactic latitudes. Important features and concentrations may be hidden by the foreground obscuration; for example, the region of the "Great Attractor," proposed by Dressler and his collaborators (e.g., Lynden-Bell et al. 1988) is intersected by the Galactic plane. The northern extension of the PerseusPisces supercluster may be also hidden by the Galactic extinction (e.g., Focardi, Marano, and Vettolani 1984). Kraan-Korteweg (1989) reviews some of the related problems. Here we report on the existence of a probable rich cluster, or a set of clusters, seen through an extinction window in the direction of the Galactic bulge.

As a byproduct of their survey for variable and propermotion stars, Terzan and his collaborators discovered several tens of diffuse objects in the general direction of the Galactic Bulge, viz., the Sagittarius bright cloud B (Terzan, Bernard, and Ju 1978; Terzan and Bernard 1978; Terzan and Ju 1980; Terzan 1985; Terzan and Ounnas 1988). The objects are all in the approximate $\alpha$ range $17^{\mathrm{h}} 04^{\mathrm{m}}-17^{\mathrm{h}} 42^{\mathrm{m}}, \delta$ range $-24^{\circ}$ to $-33^{\circ}$ (B1950). This region, centered near $l_{\mathrm{II}} \sim 358^{\circ}, b_{\mathrm{II}} \sim+7^{\circ}$, has a patchy, but obviously very heavy extinction (we estimate $A_{V} \geqslant 3^{\mathrm{m}}-4^{\mathrm{m}}$ ). These diffuse objects are a mixed bag; in the discovery papers, some were tentatively classified as galaxies, some as possible globular clusters, others as planetary nebulae. During an imaging search for obscured globular clusters, one of us (S.G.D.) obtained images of 14 of them. Out of those, two turned out to be probable globular clusters (TJ 5 and TJ 23), one a planetary

\footnotetext{
a) Based in part on the observations made at Las Campanas Observatory, as a part of a collaborative agreement between the California Institute of Technology and the Carnegie Institution of Washington.

b) Visiting Astronomer, Cerro Tololo Inter-American Observatory, NOAO, operated by AURA, Inc., under contract with the NSF

c) Alfred P. Sloan Foundation fellow.
}

nebula (TBJ 41), and the rest were obviously galaxies. A dozen of the remaining objects may be classified as galaxies from their appearance on the SRC/I sky survey films. The magnitudes of detected galaxies are typically in the range $R \sim 17^{\mathrm{m}}-18^{\mathrm{m}}$, within $10-20$ arcsec apertures, although the measurements are difficult and still very preliminary because of the heavy stellar foreground.

The occurrence of so many background galaxies in a relatively small and heavily obscured area is remarkable, and indicative of a possible extinction window in this general direction. They are probably luminous ellipticals and/or bright SO or Sp bulges: anything with a lower surface brightness would not be detected through the heavy extinction and foreground. None of the galaxies and possible galaxies are detected in the IRAS PSC, which corroborates their identification as early types. Given the heavy foreground extinction in this direction (even if there is a "window"), and the highly uncertain surface-brightness selection effects, this grouping of galaxies may be just the tip of the iceberg, signaling the presence of a larger concentration, possibly even a rich cluster. However, redshifts are necessary before it can be decided whether this is the case.

Johnston et al. (1981), and Wakamatsu and Malkan (1981) independently discovered another rich, x-ray cluster, $4 \mathrm{U}$ 1708-23, in Ophiuchus, with $l_{\mathrm{II}}=0.5^{\circ}, b_{\mathrm{II}}=+9.4^{\circ}$, at $z=0.028(c z=8400 \mathrm{~km} / \mathrm{s})$. This was noted by Terzan (1985), who in an addendum to his paper remarked on the discovery of over a hundred obscured galaxies in the area near and between the Ophiuchus cluster, and the Sagittarius Cloud B area. Some of these objects were listed subsequently by Terzan and Ounnas (1988).

Motivated by this finding, we undertook to obtain images and spectra of the Terzan et al. galaxies, and explore the possibility that there is a massive, relatively nearby (super?) cluster, hidden behind the Galactic bulge.

\section{THE OBSERVATIONS AND DATA REDUCTIONS}

We obtained CCD images and long-slit spectra for most of the diffuse Terzan et al. objects published before 1988 (i.e., 
excluding the list presented by Terzan and Ounnas 1988). The coordinates and the finding charts can be found in the original papers by Terzan et al.

A few words on the names and notation are in order; we try to keep with the prevailing usage. The four objects listed by Terzan, Bernard, and Ju (1978) and by Terzan and Bernard (1978) are denoted as TBJ, and are repeated in the list by Terzan and Ju (1980), which we denote as TJ. Following the usage in the original papers, the remaining objects are denoted as Trz, with the numbering continuing that of the TJ list: i.e., the last object listed by Terzan and Ju (1980) is TJ 24, and the first object listed by Terzan (1985) is Trz 25. These Terzan objects should not be confused with the globular clusters discovered earlier by Terzan (1971), known as Trz 1-11.

The initial spectroscopic observations were done using the Schechter Modular CCD Spectrograph on the LCO 100 in. telescope on the nights of UT 1988 June 6, 8, and 9. The detector was a TI $800 \times 800 \mathrm{CCD}$, and the grating used had 600 grooves $/ \mathrm{mm}$, giving the average dispersion of $3.05 \AA$ pixel, giving the wavelength coverage range 4670-7090 $\AA$. The spectrum of TJ 8 was obtained at the LCO $100 \mathrm{in}$. telescope on the night of UT 1988 September 13 using an almost identical instrument setup. Additional observations were performed using the Air Schmidt \#1 (red) spectrograph at the CTIO $4 \mathrm{~m}$ telescope, on the nights of UT 1988 July 14 16 . The detector was a GEC $576 \times 385 \mathrm{CCD}$, and the grating used had 400 grooves $/ \mathrm{mm}$, giving the average dispersion of $5.71 \AA /$ pixel, and the wavelength coverage range 4330-7620 $\AA$.

Spectra of the K-giant velocity standards from Yoss, Kar- man, and Hartkopf (1981), and from Abt and Biggs (1972), and spectra of $\mathrm{He}-\mathrm{Ne}$ arc lamps were obtained interspersed with the observations of Terzan et al. objects. In addition, the wavelength calibration shifts were monitored by measuring the wavelengths of the night sky emission lines (we find this to be the most reliable method). We also observed nine bright NGC galaxies with known redshifts on the night of UT 1988 July 16, in order to have an external comparison. Flux standards from Stone and Baldwin (1983) and Baldwin and Stone (1984) were used to calibrate the instrument response. The data were reduced using standard techniques. The typical heliocentric redshift corrections were $\sim 16 \mathrm{~km} / \mathrm{s}$ for the data obtained in the CTIO run, and $\sim-1 \mathrm{~km} / \mathrm{s}$ for the data obtained in the LCO run.

Direct CCD images were obtained for all objects using the CTIO 60 in. and LCO 40 in. telescopes in the same runs. In this paper we present the redshift measurements. The photometry and the velocity dispersions for galaxies, and the results on globular cluster candidates and planetary nebulae will be presented in future publications.

A few of the representative galaxy spectra are shown in Fig. 1. Two of the objects (TJ 2 and Trz 37) had foreground stars superposed, making their spectra essentially unusable. Six of the galaxies observed ( $\sim 25 \%$ ) show strong emission lines, characteristic of starburst or Seyfert 2 nuclei. Their high central surface brightness was probably an important selection effect.

For the emission-line objects, redshifts were obtained by line centering, with the highest weight given to the $\mathrm{H} \alpha$ line (because of the CCD sensitivity and the foreground extinction, as well as the strength of this line). Absorption red-

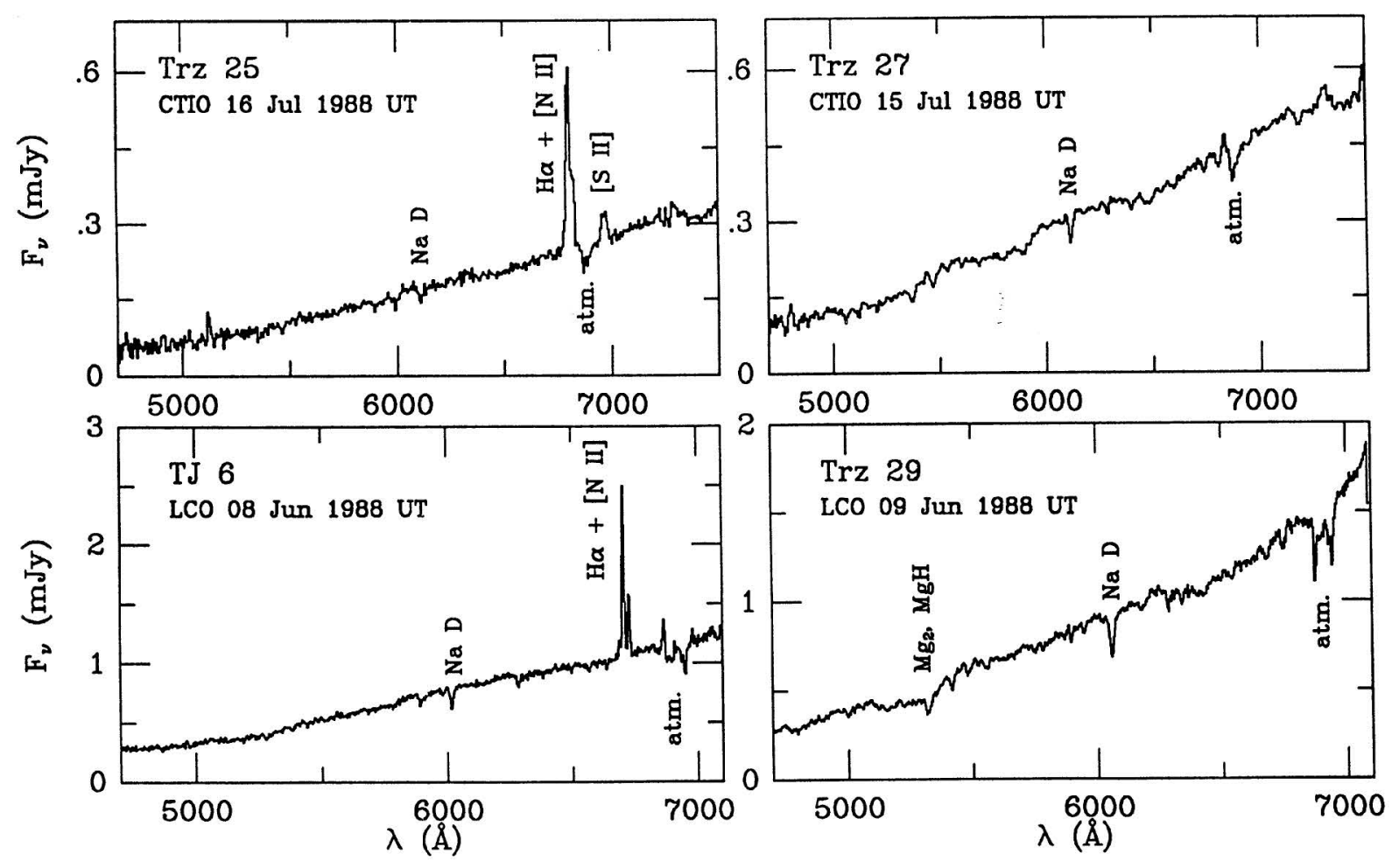

FIG. 1. Representative spectra for four objects, with line emission (left) and with pure stellar continua (right). The top two were obtained at the CTIO 4 $\mathrm{m}$, and the bottom two at the LCO $100 \mathrm{in}$. telescope. The principal spectroscopic features are labeled. 
shifts were obtained from centering of the $\mathrm{Na} \mathrm{D}$ doublet, and the cross-correlation with spectra of K-giant velocity standards. After the initial internal and external comparisons, we found discrepancies of up to $300 \mathrm{~km} / \mathrm{s}$ in measured redshifts. These were ultimately traced to the differential flexing of the spectrograph, in spite of our effort to monitor the wavelength shifts by interspersed arc lamp exposures. For the longer exposures of galaxies, but not for the short exposures of velocity standards, we could correct for such wavelength shifts to better than $50 \mathrm{~km} / \mathrm{s}$ by measuring the wavelengths of the prominent night sky emission lines. Consequently, we decided to use the redshifts obtained from the line centering only. Whereas this method has generally a lower accuracy than the cross-correlation technique, we believe that it gives more reliable results for our dataset. In order to do that, we needed to adopt effective rest-frame wavelengths for the $\mathrm{Na}$ D $5890+5896$ doublet, and for the emission-line blend of $\mathrm{H} \alpha$ and [N II] 6548,6583. These wavelengths will be affected by the intrinsic line ratios, other absorption features present, velocity dispersions in the galaxies themselves, and by our instrumental resolutions. After some experimentation, we concluded that the best choices for the effective wavelengths are $5894 \pm 0.5 \AA$ for the Na D

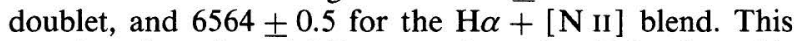
translates into the redshift uncertainty of $\sim 30 \mathrm{~km} / \mathrm{s}$, and is comparable to our rms wavelength calibration errors. We thus expect a net baseline error of $\sim 50-60 \mathrm{~km} / \mathrm{s}$ for the redshifts.

The accuracy of the redshifts were checked internally, by comparing the results for multiple exposures for a given galaxy ( taken on the same night, different nights with the same instrument, or in the two different runs), and externally, by comparing the measurements of nine NGC galaxies with the values from the CfA redshift catalog (Huchra 1989, private communication). The distributions of redshift differences are shown in Fig. 2. We find that our internal accuracy is at the level of $\sim 30 \mathrm{~km} / \mathrm{s}$, driven mostly by the errors in the wavelength calibrations. There is no significant systematic difference between our redshifts, and those from the CfA catalog, and the net scatter is at the level of $\sim 60 \mathrm{~km} / \mathrm{s}$, which is a convolution of our errors, and the typical errors quoted for the CfA values, $\sim 20 \mathrm{~km} / \mathrm{s}$. We thus conclude that our redshift errors are indeed close to $\sim 50 \mathrm{~km} / \mathrm{s}$.

The heliocentric redshifts for the galaxies are listed in $\mathrm{Ta}$ ble I. For objects where both $\mathrm{Na} \mathrm{D}$ and $\mathrm{H} \alpha$ measurements were available, average values are given. For the objects observed both at CTIO and LCO, averages for the two runs are given. Column (3) gives the observatory code, " $T$ " for CTIO, "C" for LCO, and "CT" for both. Letter "E" in column (4) indicates that line emission is present.

\section{DISCUSSION OF THE RESULTS}

Our direct images and spectra show that most of the Terzan et al. diffuse objects are indeed galaxies (about $30 \mathrm{ob-}$ jects; some are uncertain ). There are also four planetary nebulae (TJ 8, TJ 19, TBJ 4 = TJ 19, and Trz 41), one open cluster (TJ 20), two reflection nebulae (TJ 22 and TJ 24), and two or three possible globular clusters (TJ 5, TJ 23, and possibly TBJ $3=$ TJ 13 ). We note that Webbink (1985) lists TBJ $2=$ TJ 16, TJ 15, and TBJ $1=$ TJ 17 as Galactic globular clusters; these objects are very definitely background galaxies.

The galaxies span the velocity range $\sim 6200-11600 \mathrm{~km} / \mathrm{s}$, with the mean and median redshifts of 9032 and $8674 \mathrm{~km} / \mathrm{s}$
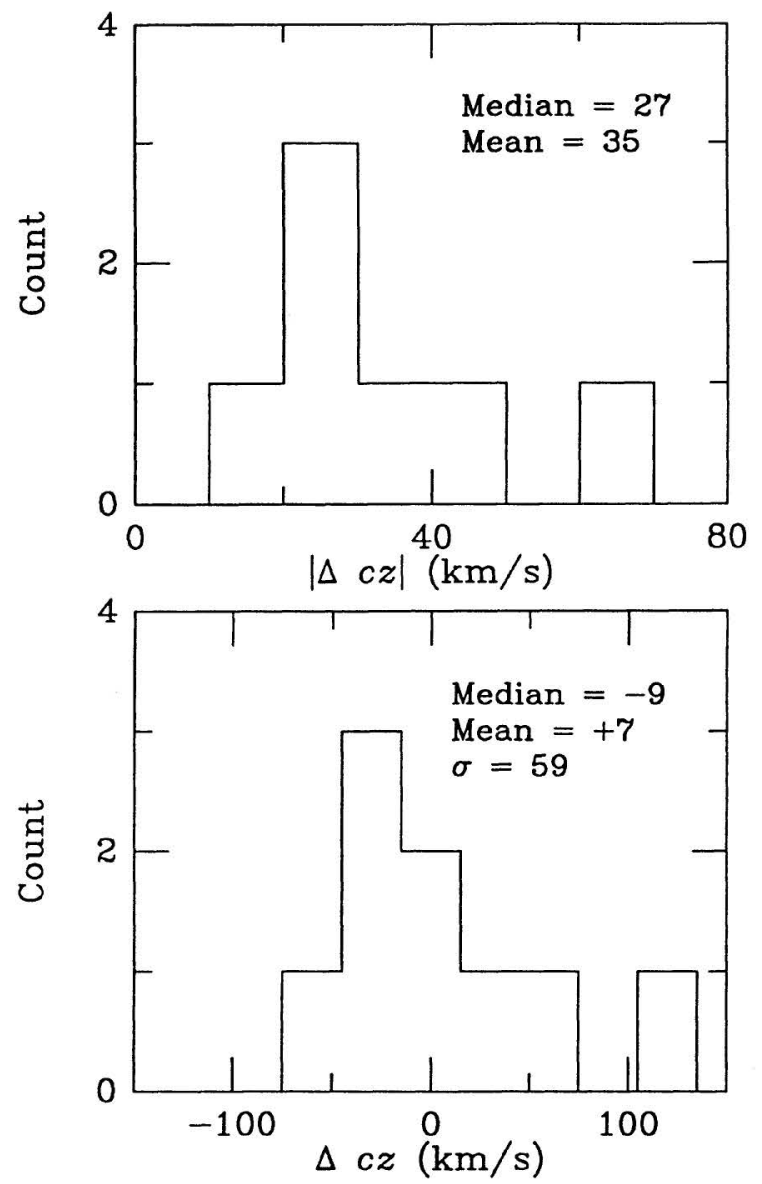

Fig. 2. Internal and external comparisons. The top histogram is for the velocity differences obtained in different exposures for the same object. The bottom one represents the heliocentric redshift differences for nine NGC galaxies taken from the CfA redshift catalog. The basic statistics are indicated.

TABLE I. Redshifts for the Sagittarius galaxies.

\begin{tabular}{|c|c|c|c|}
\hline $\begin{array}{c}\text { Galaxy } \\
(1)\end{array}$ & $\begin{array}{c}c z_{\odot} \\
(\mathrm{km} / \mathrm{s}) \\
(2)\end{array}$ & $\begin{array}{l}\text { Obs. } \\
(3)\end{array}$ & $\begin{array}{c}\text { Em.? } \\
(4)\end{array}$ \\
\hline $\begin{array}{l}\text { TJ 1 } \\
\text { TJ } 3 \\
\text { TJ 6 } \\
\text { TJ 14 } \\
\text { TJ 15 } \\
\text { TJ 17= TBJ 1 } \\
\text { Trz 25 } \\
\text { Trz 26 } \\
\text { Trz 27 } \\
\text { Trz 28 } \\
\text { Trz 29 } \\
\text { Trz 30 } \\
\text { Trz 31 } \\
\text { Trz 32 } \\
\text { Trz 33 } \\
\text { Trz 34 } \\
\text { Trz 35 } \\
\text { Trz 36 } \\
\text { Trz 38 } \\
\text { Trz 39 } \\
\text { Trz 40 }\end{array}$ & $\begin{array}{r}8824 \\
9143 \\
6259 \\
8674 \\
8619 \\
6270 \\
11086 \\
11282 \\
11355 \\
11525 \\
8330 \\
11422 \\
8923 \\
8809 \\
7664 \\
8566 \\
9406 \\
8478 \\
8101 \\
8525 \\
8406\end{array}$ & $\begin{array}{l}\mathrm{T} \\
\mathrm{T} \\
\mathrm{CT} \\
\mathrm{CT} \\
\mathrm{T} \\
\mathrm{C} \\
\mathrm{T} \\
\mathrm{CT} \\
\mathrm{T} \\
\mathrm{T} \\
\mathrm{C} \\
\mathrm{T} \\
\mathrm{T} \\
\mathrm{T} \\
\mathrm{T} \\
\mathrm{CT} \\
\mathrm{T} \\
\mathrm{T} \\
\mathrm{T} \\
\mathrm{T} \\
\mathrm{C}\end{array}$ & $\begin{array}{l}\mathrm{E} \\
\mathrm{E} \\
\mathrm{E}\end{array}$ \\
\hline
\end{tabular}


respectively. The histogram of the heliocentric redshifts is shown in Fig. 3. There appear to be two concentrations, the main one near $8600 \mathrm{~km} / \mathrm{s}$ (14 galaxies in this sample, or $67 \%$ ), and a possible one near $11340 \mathrm{~km} / \mathrm{s}$ (five galaxies, or $24 \%$ ). Whereas the expected distribution of redshifts for a uniform distribution of galaxies in this region is virtually impossible to construct, due to the unknowns in the foreground extinction, selection effects, etc., these results strongly suggest that there is a concentration in the redshift range $\sim 7600-9400 \mathrm{~km} / \mathrm{s}$, which we interpret as the signature of a possibly rich cluster. At this time, we cannot judge the statistical significance of the apparent clump at 11340 $\mathrm{km} / \mathrm{s}$.

Using the Balmer decrement measurements for the emission-line galaxies in the sample, we derive for the total lineof-sight extinction to these objects $E_{B-V}=1.4$, or $A_{V}$ $=4.4 \mathrm{mag}$ (for $\operatorname{Trz} 25$ and $\operatorname{Trz} 28$ ), and $E_{B-V}=2.0$, or $A_{V}$ $=6.3 \mathrm{mag}$ (for TJ 1 and TJ 6). These estimates may be uncertain by as much as $\Delta A_{V} \sim 1 \mathrm{mag}$, and include any possible internal extinction in the galaxies themselves. The differences probably reflect the patchiness of the foreground Galactic extinction. The implied correction to the galaxy magnitudes ( $\left.R_{\mathrm{obs}} \sim 17\right)$ makes them more or less typical for their Hubble distances, if these objects are luminous ellipticals or early-type spirals.

The average of the mean and the median positions for the 14 galaxies in the redshift range $\sim 7600-9400 \mathrm{~km} / \mathrm{s}$ is: $\langle\alpha\rangle=17^{\mathrm{h}} 10^{\mathrm{m}} 35^{\mathrm{s}},\langle\delta\rangle=-25^{\circ} 07^{\prime} 40^{\prime \prime}$ (B1950), or in the Galactic system: $\left\langle l_{\mathrm{II}}\right\rangle=359.13^{\circ},\left\langle b_{\mathrm{II}}\right\rangle=+8.04^{\circ}$. The mean and the median redshifts are 8605 and $8593 \mathrm{~km} / \mathrm{s}$, respectively. The velocity dispersion of this clump is 430 $\mathrm{km} / \mathrm{s}$, which is typical for Abell clusters of richness class 0 1. However, given the uncertainties of the foreground extinction and the selection effects, we cannot make any worthwhile estimates about the overall size, mass, or richness of the putative Sagittarius cluster at this time.

The question arises as to whether this grouping of galaxies is a cluster on its own, or just an extension of the previously discovered Ophiuchus cluster. The Sagittarius concentration is some $3^{\circ}$ away from the center of the Ophiuchus cluster, whose position is well defined by the $\mathrm{x}$-ray maximum (Johnston et al. 1981). At $c z \simeq 8600 \mathrm{~km} / \mathrm{s}$, this separation corresponds to $\sim 4 h{ }_{100}^{-1} \mathrm{Mpc}$, which is comparable to the mean intercluster separations in high-density superclusters (e.g., Bahcall and Soneira 1984). On the other hand, Abell radii of the Coma and A1367 clusters moved to that redshift would be $\sim 1^{\circ}$. We thus tentatively conclude that the proposed Sagittarius cluster is an entity separate from the Ophiuchus cluster, but that the two may well be physically associated in a larger structure.

Whereas the selection effects due to the foreground extinction are very hard to estimate, they must be highly significant, and we have probably seen only a small fraction of the galaxies in this area. If this is the case, we may be dealing with a massive system, possibly a supercluster, almost directly behind the Galactic Bulge, and slightly above the Ga-

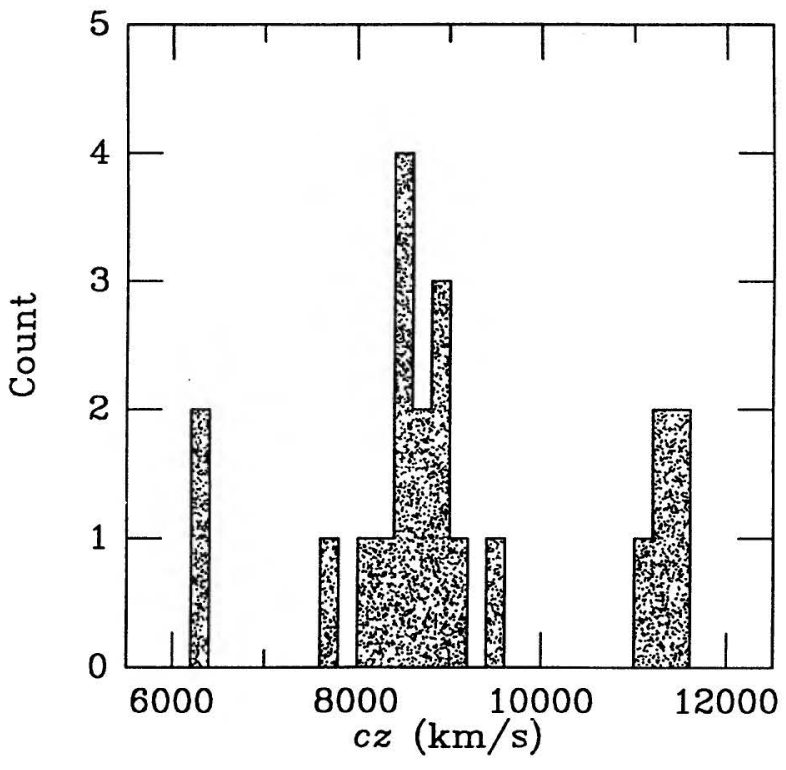

FIG. 3. The heliocentric redshift histogram for the galaxies in our sample. Note the prominent concentration at $c z \sim 8600 \mathrm{~km} / \mathrm{s}$, and another one at $c z \sim 11300 \mathrm{~km} / \mathrm{s}$. No galaxies were found outside the redshift limits of this graph.

lactic plane. This putative Sagittarius-Ophiuchus supercluster may be an important factor in the local supergalactic dynamics, possibly even more massive than the ComaA1367 system, which is at roughly the same distance. It may be an obscured part of the "Great Wall" structure, recently discovered by Geller and Huchra (1989). Observations of the remaining galaxies from the list by Terzan and Ounas (1988), and a systematic search for more galaxies in this area could clarify the issue.

Other important mass concentrations may well have escaped unnoticed so far. A careful and systematic examination of the "zone of avoidance," using the optical sky surveys, or a follow-up to the IRAS and Rosat all-sky surveys, is both feasible and desirable. An example may be the study of low-latitude IRAS galaxies by Dow et al. (1988).

We would like to thank the staff of Cerro Tololo and Las Campanas Observatories for their help during our observing runs, and to S. Morris and S. Anderson for obtaining the spectrum of $\mathrm{TJ}$ 8. The paper benefited from the constructive criticism by Matt Malkan, the referee. This work was supported in part by the Alfred P. Sloan Foundation and by California Institute of Technology (S.D.), NSF Grant No. AST 87-21705 (J.R.M.), and by a Kingsley fellowship (D.J.T.) R.R.dC. is on leave of absence from Observatorio Nacional, CNPq, Rio de Janeiro, Brazil.

REFERENCES

Abt, H., and Biggs, E. (1972). Bibliography of Stellar Radial Velocities (Latham Process Corp., New York).

Bahcall, N., and Soneira, R. (1984). Astrophys. J 277, 27.

Baldwin, J., and Stone, R. (1983). Mon. Not. R. Astron. Soc. 204, 347.
Dow, M., Lu, N., Houck, J., Salpeter, E., and Lewis, B. (1988). Astrophys. J. Lett. 324, L51.

Focardi, P., Marano, B., and Vettolani, P. (1984) . Astron. Astrophys. 136, 178. 
Geller, M., and Huchra, J. (1989). Science 246, 897.

Johnston, M., Bradt, H., Doxsey, R., Margon, B., Marshall, F., and Scwartz, D. (1981). Astrophys. J. 245, 799.

Kraan-Korteweg, R. (1989). Rev. Mod. Astron. 2, 119.

Lynden-Bell, D., Faber, S., Burstein, D., Davies, R., Dressler, A., Terlevich, R., and Wegner, G. (1988). Astrophys. J. 326, 19.

Stone, R., and Baldwin, J. (1983). Mon. Not. R. Astron. Soc. 206, 241.

Terzan, A. (1971). Astron. Astrophys. 12, 477 (Erratum in ibid. 15, 336).

Terzan, A. (1985). ESO Messenger No. 42, 4.

Terzan, A., and Bernard, A. (1978). ESO Messenger No. 15, 14.
Terzan, A., Bernard, A., and Ju, K. (1978). C. R. Acad. Sc. Paris 287B, 157.

Terzan, A., and Ju, K. (1980). ESO Messenger No. 20, 6.

Terzan, A., and Ounnas, Ch. (1988). Astron. Astrophys. Suppl. Ser. 76, 205.

Wakamatsu, K., and Malkan, M. (1981). Publ. Astron. Soc. Jpn. 33, 57. Webbink, R. (1985). In Dynamics of Star Clusters, IAU Symposium No. 113, edited by J. Goodman and P. Hut (Reidel, Dordrecht), p. 541

Yoss, K., Karman, R., and Hartkopf, W. (1987). Astron. J. 86, 37. 